\title{
A NEW METHOD FOR MARKING ANT LARVAE
}

\author{
By JAMES F. HARE ${ }^{1}$ \\ Erindale College, University of Toronto \\ Mississauga, Ontario, Canada L5L 1C6
}

In most ethological studies, an observer must recognize individual animals or at least certain classes of animals in order to acquire meaningful information. Individual animals are distinguished either by morphological differences or through the application of a distinguishing mark by the investigator.

In recent studies conducted by Hare (1987) and Alloway \& Hare (under editorial review) where brood of a slave-making ant species (Harpagoxenus americanus) and host species (Leptothorax longispinosus) were offered simultaneously to host species workers, an observer was required to identify the species of any given larva quickly and reliably. Species identification of ant larvae has traditionally relied upon one or more of the following morphological characteristics: general body shape, mandible shape, pattern, structure and abundance of hairs, location and abundance of integumentary spinules, head shape, dentition of the mandibles and physical characteristics of other mouthparts (see Wheeler \& Wheeler, 1960). None of these characteristics are useful in distinguishing Harpagoxenus from Leptothorax larvae (Wheeler \& Wheeler, 1960; Hare, personal observation). Thus for our research, we required a technique whereby larvae could be given a visible mark that would not bias the acceptance of marked versus unmarked larvae.

Several techniques have been developed for marking adult ants (see Stuart 1986 for a review). Techniques reported for marking brood either damage the brood (e.g. filling eviscerated larval skins with coloured gelatine; Brian 1975), or the marks do not persist because of grooming by adult workers [e.g. spots of of coloured wax applied to larval cuticle (Brian 1975), bits of coloured plastic glued

'Present Address: Department of Zoology, University of Alberta, Edmonton, Alberta, Canada T6G 2E9

Manuscript received by the editor November 20, 1988. 
to the larval cuticle (Isingrini et al. 1985) or coloured spots of paint applied to cocoons (R. J. Stuart, personal communication) ]. Other techniques, although effective, are not efficient in terms of the time required to obtain marked larvae. For example, Brian \& Rigby (1978) and Cole (1981) report that Sudan black, an oil soluble dye, fed to a queen results in the production of coloured eggs. Apparently, this mark is retained through all life stages including adulthood (R. J. Stuart, personal communication). For the purposes of our experiments, waiting for dye to be ingested by the queen and for the marked eggs to develop into larvae was inefficient.

Adult ants are known to devour even slightly damaged brood rapidly and to regurgitate this material to healthy larvae (Wilson, 1971). Thus it seems plausible that if a coloured substance is applied externally to brood that causes workers to perceive marked brood as damaged, the coloured substance will end up inside healthy larvae in the colony. Such a mark would be visible to a human observer through the translucent larval cuticle, and if effective would provide a rapid and inexpensive means of marking large numbers of larvae. Here I describe a technique for obtaining marked larvae, present data on the persistence of the mark, and demonstrate that marking does not bias larva acceptance by Leptothorax ambiguus or L. longispinosus workers.

\section{Methods ANd Materials}

Nests of both L. ambiguus and L. longispinosus were collected during September, October and November 1985 and from April through July 1986 at various sites within the regional municipalities of Halton and Peel, Ontario, Canada. Ant colonies were cultured in the laboratory employing techniques described by Alloway (1979) and Hare (1987).

In a pilot study, 10 larvae smeared with Testor's red enamel paint (\#1150, commonly used to paint plastic models and available at local hobby retailers) were placed inside the nest of each of $17 \mathrm{~L}$. longispinosus colonies. Within $24 \mathrm{~h}, 5$ to $10(8.5 \pm 1.87$ [mean $\pm \mathrm{SE}$ ] $)$ of those larvae were cannibalized by adult workers. During this $24 \mathrm{~h}$ period, trophallactic exchange of food from workers to previously unmarked larvae resulted in a large proportion $(0.63 \pm 0.17$ $[$ mean $\pm \mathrm{SE}])$ of each colony's larvae becoming marked internally 
with the paint. Between $31.5 \%$ and $85.7 \%$ of the previously unmarked larvae became marked internally, thus providing a minimum of 6 and a maximum of 34 marked larvae in the test colonies. The colour red was selected for use in all experiments because ants are not sensitive to light of this wavelength (Wilson, 1971), thus reducing the possibility of any visually mediated bias in larva acceptance.

To test for any influence of the mark on larva acceptance, an experiment was performed. Internally marked larvae were obtained by covering the mouthparts and at least $50 \%$ of the cuticle of several L. ambiguus larvae with Testor's red enamel and placing these larvae inside an $L$. longispinosus nest. Adults of the recipient colony cannibalized these larvae and in most cases fed this material to their own larvae. Allospecific larvae were used to 'transmit' the mark since they are less likely to be accepted for tending (see Hare and Alloway, 1987) and are thus more likely to be cannibalized.

On the next day, worker groups were established by choosing three L. longispinosus workers arbitrarily from each of 13 colonies and placing each group in a new culture dish with a nest, water vial and food (see Hare and Alloway 1987). Groups were allowed 24 hours to explore their new environment prior to testing.

Since Plateaux (1960) found that Leptothorax nylanderi workers could only distinguish between conspecific larvae and Solenopsis fugax larvae when conspecific larvae were present, larvae of the two 'types' were offered simultaneously to the workers. Under this protocol, a single larva of each 'type' (marked and unmarked) were placed on a clean glass coverslip and positioned immediately in front of the nest entrance. Within each pair, larvae were matched visually for size and placed as close together as possible, (but not in physical contact) in the centre of the coverslip. Workers were allowed to retrieve one larva into their nest, their choice was recorded, and a new pair of larvae was presented. Paired presentations of this sort continued until the supply of either marked or unmarked larvae for a given donor colony was exhausted. For the 13 worker groups (replicates) the number of initial paired presentations ranged from one to $12(8.2 \pm 0.8$ presentations [mean $\pm S E$ ], mode $=10$ presentations). Since each worker group was derived from a separate maternal colony, replicates were independent and the difference in the number of the two types of larvae accepted in 
paired presentations across groups can be analysed using the Wilcoxon paired sample test (Zar 1974). Occasionally more than one ant was involved in the retrieval of larvae, and in some instances both larvae were judged to be taken simultaneously. For the purpose of analysis those ties were omitted and the number of presentations for that group consequently reduced.

Following the series of paired presentations, marked and unmarked larvae which had not been taken were re-introduced in one large pile on a glass coverslip outside the nest entrance. The amount of time taken to retrieve the remaining larvae was recorded (if time allowed on the day of the trial) and the number of each type of larva remaining in the nest was recorded daily until the internal mark could no longer be distinguished (6 to 16 days). For each day, data were analysed using a heterogeneity Chi-Square test (Zar 1974) that compared the observed number of each type of larva in the nest tc an expected null difference within each group (i.e. if no preference were exhibited then we would expect equal numbers of the two types inside the nest) and for the pooled difference across groups.

The experiment was replicated using $16 \mathrm{~L}$. ambiguus worker groups and larvae from their own maternal colonies. For L. ambiguus, the number of initial paired presentations ranged between five and $17(9.0 \pm 1.1$ days [mean $\pm \mathrm{SE}])$. Data from these presentations are included and analysed as outlined above. However, the marked larvae used in these trials were marked seven to 10 days $(7.7 \pm 0.9$ [mean $\pm \mathrm{SE}]$ ) in advance of the trials (i.e. these were marked for a pilot study addressing the feasibility of obtaining marked larvae). Consequently the record of the number of marked and unmarked larvae remaining in the nest became increasingly confounded over time as the internal mark faded or disappeared. This trend became noticeable on the third day of observations and thus for L. ambiguus data are only presented on acceptance over time for the first two days.

\section{RESULTS}

Reliable data on the persistence of the internal mark are available only for the larvae offered to L. longispinosus workers in the "mark bias" experiment. In those trials, the mark duration ranged from six to 16 days $(11.1 \pm 1.0$ days $[$ mean $\pm \mathrm{SE}$ ], mode $=12$ days $)$ across groups. 
In the series of simultaneous presentations neither $L$. ambiguus nor L. longispinosus worker groups showed a significant preference for retrieving marked or unmarked larvae $(\mathrm{T}=32.5, P>0.50$ and $\mathrm{T}=37, P>0.50$ for $L$. ambiguus and $L$. longispinosus respectively). An average of $4.7 \pm 0.77$ (mean $\pm 1 \mathrm{SE}$ ) marked and $4.3 \pm 0.52$ unmarked larvae were retrieved first by the $16 \mathrm{~L}$. ambiguus groups while $4.3 \pm 0.49$ marked and $3.6 \pm 0.51$ unmarked larvae were retrieved first by the 13 L. longispinosus worker groups (Fig. 1, day 0). Furthermore, the number of groups accepting a greater number of marked larvae; unmarked larvae, or equivalent numbers of both types in paired presentations (Table I) was similar across the two species $\left(3 \times 2\right.$ contingency table analysis (Zar 1974), $x^{2}=1.1659$, $P>0.25)$.

For both L. ambiguus and L. longispinosus, the data from individual worker groups were homogeneous on each day of observation and no significant preference for either marked or unmarked larvae was shown by any single group over the course of the study (all $P>.25$, see Appendix B-1 in Hare, 1987). Similarly, no significant differences in the number of marked and unmarked larvae remaining in the nests of either species were detected when data were pooled by day (all $P>.50$, see Appendix B-1 in Hare, 1987). The mean numbers of marked and unmarked larvae accepted by worker groups on any given day were similar, although at almost all time intervals the average number of marked larvae per nest was slightly higher than that for unmarked larvae (Fig. 1).

The large increase in the mean number of marked and unmarked larvae accepted for groups of both species between day 0 and day 1 reflects the fact that the day 0 data include only those larvae retrieved first in the series of paired presentations. Following this series of presentations, 'non-preferred' larvae were re-introduced and overall, $99.3 \%$ of the marked larvae and $97.2 \%$ of the unmarked larvae available to the L. ambiguus worker groups were accepted by day 1 while $98.3 \%$ of the marked larvae and $100 \%$ of the unmarked larvae available were accepted by $L$. longispinosus worker groups during the same period. Thus, even those larvae that were 'nonpreferred' in a simultaneous choice situation were acceptable to Leptothorax workers.

The increase in the mean number of marked larvae in the $L$. longispinosus nests between days 14 and 15 (Fig. 1) is a result of the 
I. ambiguus $(\mathrm{N}=16)$

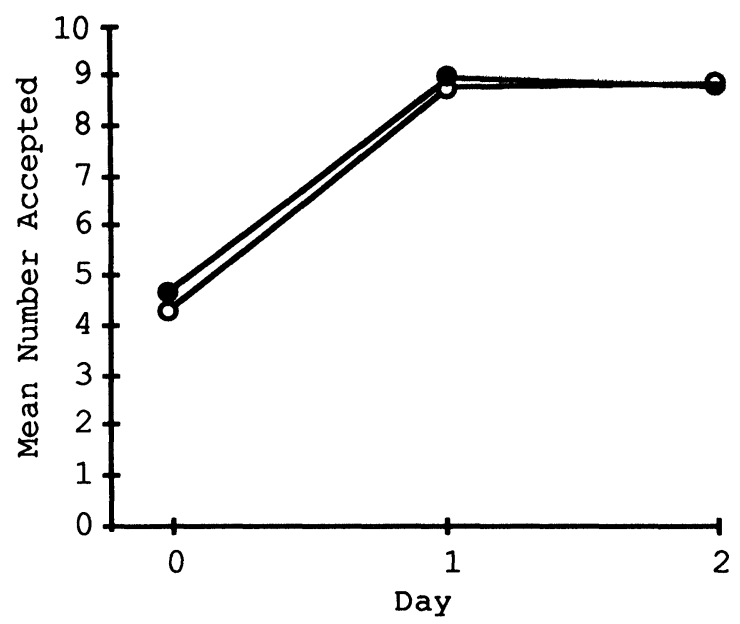

I. longispinosus

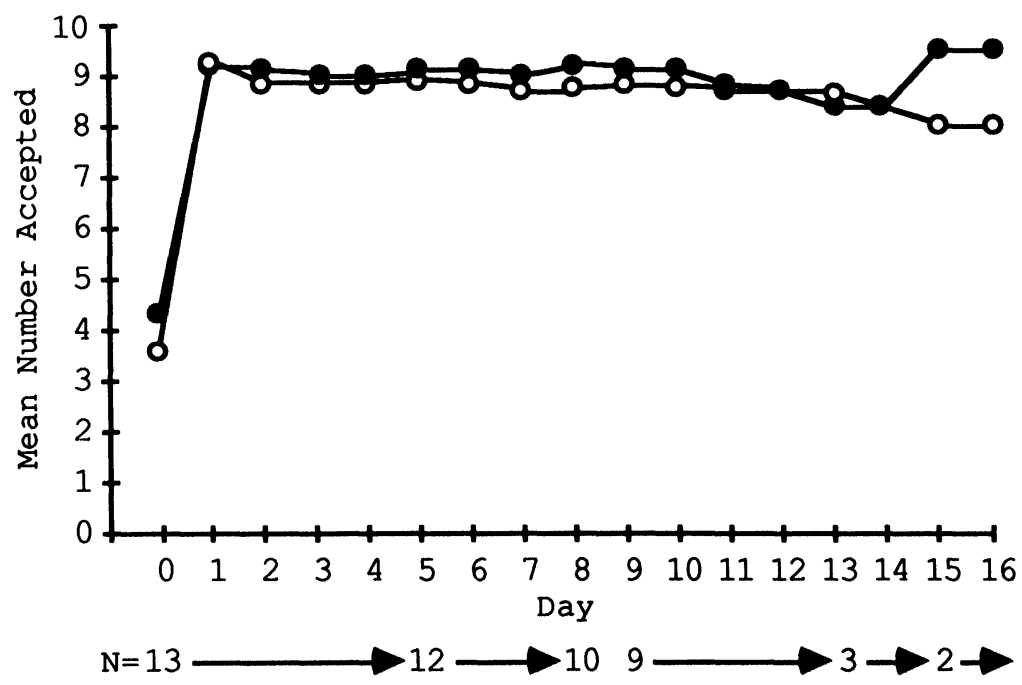

Figure 1: Mean number of marked larvae (solid circles) and unmarked larvae (open circles) accepted by Leptothorax worker groups over time. [Note Day $0=$ number accepted upon termination of paired presentations]. 
Table 1. Initial preference profiles of Leptothorax worker groups.

\begin{tabular}{|c|c|c|}
\hline & \multicolumn{2}{|c|}{ Worker Species } \\
\hline & L. ambiguus & L. longispinosus \\
\hline Groups accepting more marked larvae & 7 & 8 \\
\hline $\begin{array}{l}\text { Groups accepting more unmarked larvae } \\
\text { Groups accepting equal numbers of }\end{array}$ & 6 & 4 \\
\hline both types of larvae & 3 & 1 \\
\hline
\end{tabular}

reduced sample size and does not reflect a change in the number of larvae in the nests of the remaining groups (see Appendix B-1 in Hare, 1987). For the two L. longispinosus groups that were observed on the sixteenth day, $95 \%$ of the marked larvae and $80 \%$ of the unmarked larvae that were initially available remained intact within the nest. Without exception, marked and unmarked larvae were placed in a common brood pile within the nests of both species throughout the experiment.

\section{Discussion}

The introduction of larvae marked externally with Testor's paint provides an inexpensive and reliable technique through which large numbers of internally marked larvae can be obtained rapidly $(<24 \mathrm{~h})$. Fading of the mark was perceivable in some groups as early as six days after marking, but remained visible in others to a maximum of sixteen days. Thus, this technique would not be useful in situations requiring an extended mark duration, but its utility would ultimately depend on the specific rate of trophallactic food exchange of the ants in question. The technique should also be limited to use with small worker groups since high rates of trophallaxis between larvae and workers in full colonies can lead to contamination of unmarked larvae with the mark (Hare, unpublished data). However, this would not present a problem over the short term since "contaminated" larvae possess a very faint mark relative to those introduced as "marked" larvae in the first place (Hare, personal observation). For the purposes of our experiments with Leptothorax, the mark duration was more than adequate and there were no instances of contamination in any trial using groups of three workers.

No significant preference for marked or unmarked larvae occurred at any time interval and overall neither $L$. ambiguus nor $L$. 
longispinosus showed a preference on any day for marked or unmarked larvae. Furthermore, ants placed marked and unmarked larvae in a common brood pile within the nest. Thus the presence or absence of the mark itself did not influence larva-acceptance by Leptothorax.

Throughout the course of the experiment, deviations from equivalent numbers of marked and unmarked larvae accepted typically were in the direction of a greater number of marked larvae within both species. However, those deviations were quite small. The explanation for this pattern lies in the observed tendency of Leptothorax workers to prefer large larvae (Hare 1987). Whenever larvae were marked, it appeared that large larvae became marked more readily than small larvae. This in itself suggests that large larvae receive preferential treatment (in terms of regurgitation from adults) in their maternal colonies and results in uneven size distributions of marked and unmarked larvae. In spite of efforts to match larvae as closely as possible on the basis of size, marked larvae were often slightly larger than the unmarked larvae used, thus explaining any apparent trend towards preferential acceptance of marked larvae.

\section{SumMary}

A technique is described whereby a visually observable mark can be transmitted to the gut contents of ant larvae. The technique is inexpensive, provides large numbers of marked larvae on demand, and is useful in situations requiring a mark duration of less than 10 days. Results of a choice experiment demonstrate that the mark itself does not bias larva acceptance by Leptothorax workers.

\section{ACKNOWLEDGMENTS}

Funding for this research was provided by the Natural Sciences and Engineering Research Council of Canada in the form of a grant to T. M. Alloway and a post-graduate scholarship to J. F. Hare. I thank Lew Holowaty, Andrew Kajioka, Alikeshs Leighl and Roger Mosquera for technical assistance and T. M. Alloway and R. J. Stuart for enlightening discussions during the development of this technique. I also thank Karl Larsen, R. B. MacWhirter, J. O. Murie and P. J. Young for helpful comments on earlier drafts of this manuscript. 


\section{REFERENCES}

Alloway, T. M. 1979. Raiding behaviour of two species of slave-making ants, Harpagoxenus americanus (Emery) and Leptothorax duloticus Wesson (Hymenoptera: Formicidae). Animal Behaviour, 27: 202-210.

Alloway, T. M. \& HARE, J. F. (under editorial review in Behaviour). Experience independent attraction to slave-maker ant larvae in host-species ant workers (Leptothorax longispinosus; Hymenoptera: Formicidae).

Brian, M. V. 1975. Larval recognition by workers of the ant Myrmica. Animal Behaviour, 23: 745-756.

Brian, M. V. \& RigBY, C. 1978. The trophic eggs of Myrmica rubra L. Insectes Sociaux, 25: 89-110.

ColE, B. J. 1981. Dominance hierarchies in Leptothorax ants. Science, 212: 83-84.

HARE, J. F. 1987. Brood discrimination by leptothoracine ants (Hymenoptera: Formicidae). M. Sc. Thesis, University of Toronto.

Hare \& Alloway. 1987. Early learning and brood discrimination in leptothoracine ants (Hymenoptera: Formicidae). Animal Behaviour 35: 1720-1724.

ISINGRINI, M., LenOIR, A. \& JAIsSON, P. 1985. Preimaginal learning as a basis of colony-brood recognition in the ant Cataglyphis cursor. Proceedings of the National Academy of Sciences U.S.A., 82: 8545-8547.

Plateaux, C. 1960. Adoptions expérimentales de larves entre des fourmis de genres différents I: Leptothorax nylanderi Förster et Solenopsis fugax Latreille. Insectes Sociaux, 7: 163-170.

StUART, R. J. 1986. Use of polyester fibers to mark small leptothoracine ants (Hymenoptera: Formicidae). Journal of the Kansas Entomological Society, 59: 566-568.

Wheeler, G. C. \& Wheeler, J. 1960 . The ant larvae of the subfamily Myrmicinae. Annals of the Entomological Society of America, 53: 98-110.

Wilson, E. O. 1971. The Insect Societies. Cambridge, Massachusetts: The Belknap Press of the Harvard University Press. 548 pp.

ZAR, J. H. 1974. Biostatistical Analysis. Englewood Cliffs, New Jersey: PrenticeHall. 620 pp. 

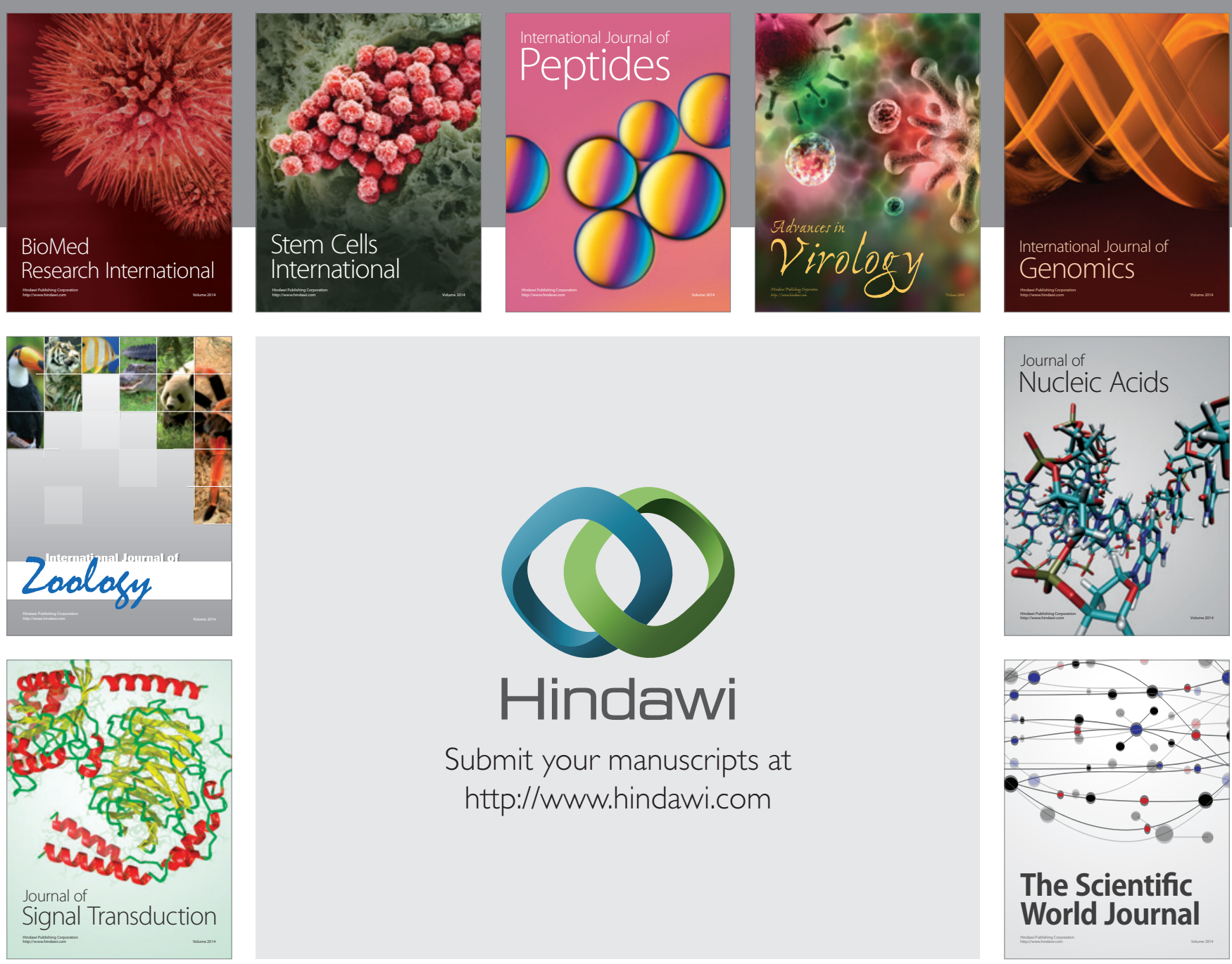

Submit your manuscripts at

http://www.hindawi.com
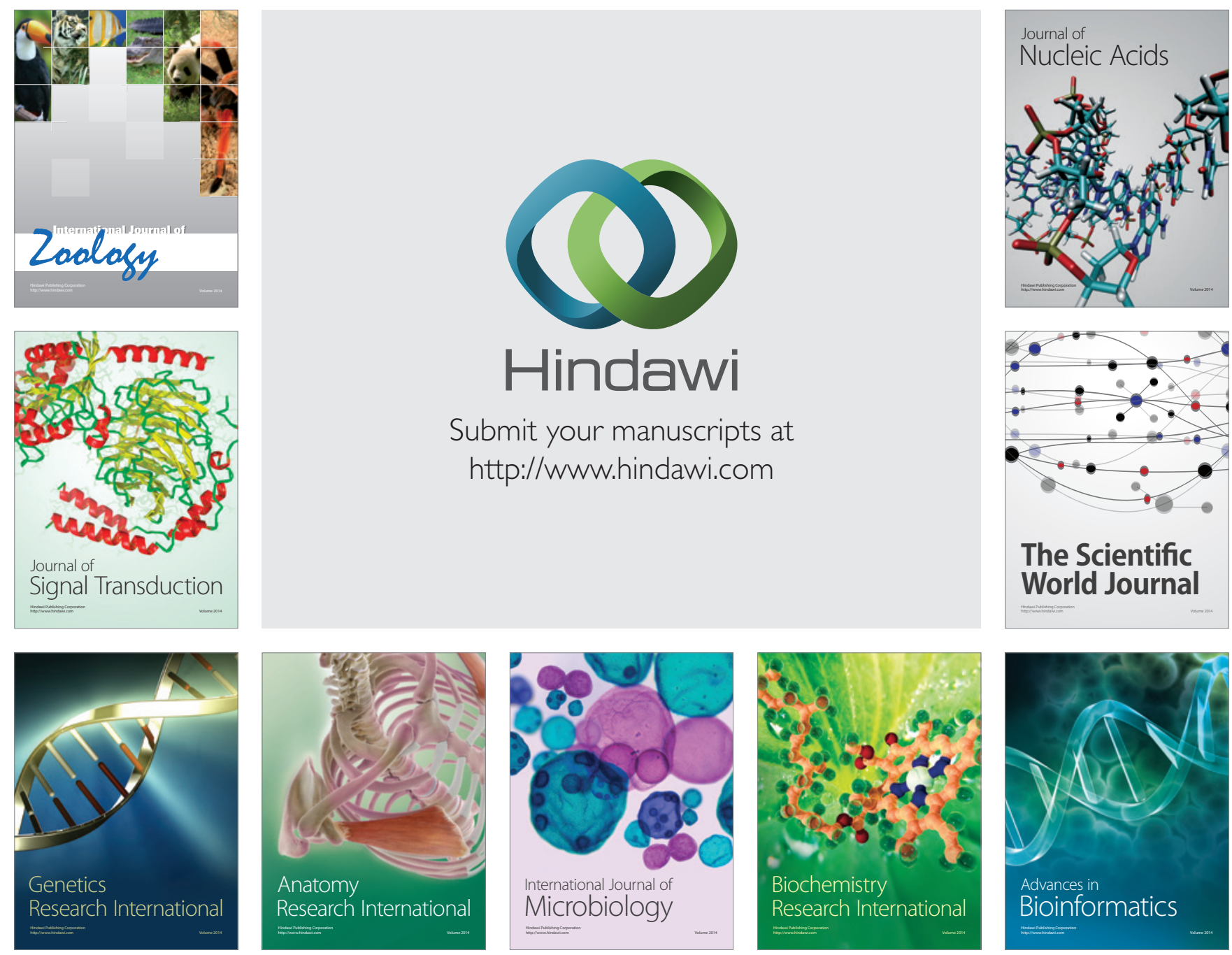

The Scientific World Journal
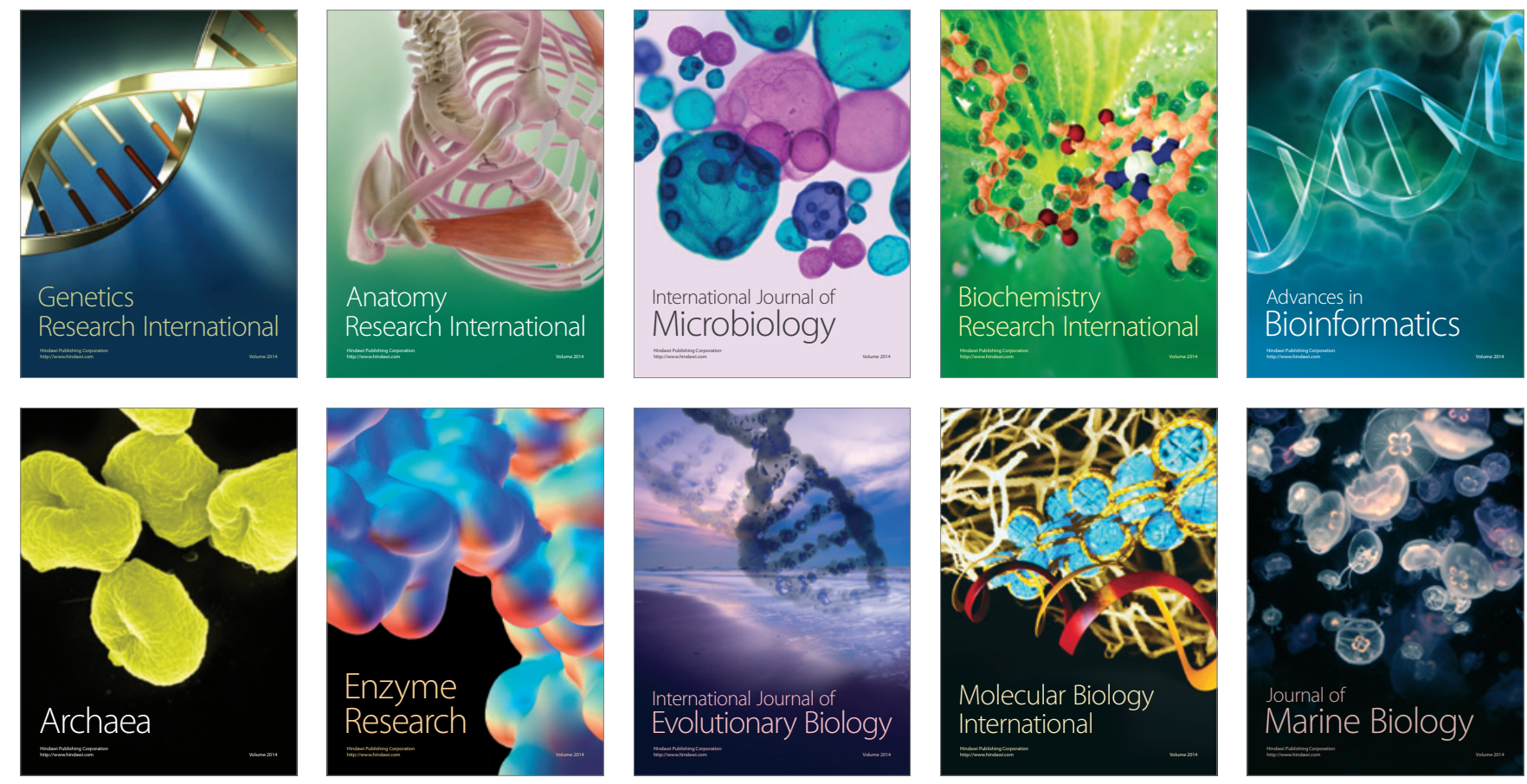